УДК $811.133 .3 ' 367$

ДОИ https://doi.org/10.18485/primling.2016.17.16

\title{
Mariana Pitar
}

\author{
Université de l'Ouest de Timişoara
}

\section{LA STRUCTURE MODULAIRE DU TEXTE PRESCRIPTIF. RÔLE ET MODALITÉS}

Le texte prescriptif, appelé aussi injonctif ou procédural en fonction de la perspective envisagée, comprend quelques genres textuels bien connus, parmi lesquels la recette de cuisine et les modes d'emploi. Avec un parcours historique assez important, la recette de cuisine passe d'un texte compacte, mélangé à la narration, à un texte de plus en plus structuré, organisé en parties distinctes. D'une date plus récente, les modes d'emploi semblent être arrivés assez vite à une structure claire, organisée en modules, dont les informations sont transmises par deux codes sémiotiques - le texte et les images (dessins, schémas, symboles etc.).C'est ce type d'organisation textuelle modulaire que nous nous proposons de présenter dans notre communication. En nous appuyant sur l'exemple de ces deux genres mentionnés nous allons étudier la structure compositionnelle de ces textes qui est le reflet textuel de leur structure sous-jacente commune à tous les genres du texte prescriptif: objectif proposé - état initial - opérations - état final-objectif réalisé. Cette macrostructure textuelle organise le texte en modules, chacun avec un rôle précis sur les plans sémantiques, syntaxique et compositionnelle, manifestés à la surface textuelle d'une manière évidente.

Ce qui est aussi important c'est le fait que cette structure détermine le choix de certaines modalités, différentes au niveau de chaque module, qui s'expriment par des marqueurs de modalité qui passent du niveau linguistique (modes des verbes, valeur sémantique des mots etc.) au niveau extralinguistique (images, position du texte dans la page, symboles etc.). Nous allons les étudier en stricte liaison avec la structure modulaire de ce type de texte.

Mots-clés : texte prescriptif, texte injonctif, genre textuel, modalité, structure sous-jacente, macrostructure textuelle, recette de cuisine, mode d'emploi, modalité prescriptive

\section{Présentation générale du texte prescriptif}

Le texte prescriptif, connu surtout en linguistique comme texte injonctif, est un texte qui se différencie des autres types par des caractéristiques tout à fait particulières, surtout par son aspect pratique, par son but qui vise la réalisation d'une activité concrète. On peut ainsi considérer ce texte comme un intermédiaire sémiotique, une interface textuelle entre deux activités pratiques : une, réalisée par un auteur-connaisseur qui la transforme en texte et l'autre réalisée par le destinataire, selon les instructions données par l'intermédiaire du texte. Cette relation peut être représentée, d'un point de vue cognitif, par le schéma suivant : 
auteur (encodeur de l'activité en texte) - texte - destinataire (décodeur du texte et réalisateur de l'activité)

Pour exprimer le caractère pratique spécifique de ce texte nous rappelons aussi la théorie de Bronckart (1997) qui classifie les textes selon les mondes dont ils font partie. Il y a ainsi deux mondes, chacun donnant une catégorie spécifique de textes :

- le monde ordinaire, des hommes (ou le monde réel); selon ce critère il y a des textes de l'ordre d'exposer;

- le monde discursif (ou le monde imaginaire) qui produit des textes de l'ordre de raconter

Selon ces critères le texte injonctif fait partie des types de texte de l'ordre d'exposer et du monde ordinaire, ce qui exprime encore une fois son lien avec l'activité pratique et le monde réel. Cette caractéristique se reflète dans tous les aspects textuels spécifiques : réseau sémantique, lexico-grammatical, structure compositionnelle.

La définition la plus appropriée du texte injonctif nous semble celle de Vigner (apud J.M. Adam $1992: 95)$ :

«... la représentation d'une transformation d'état que le lecteur aura à réaliser sur injonction du scripteur, transformation qui s'accomplira par le moyen d'un algorithme revêtant la forme d'activités gestuelles programmées selon une logique technique (et culturelle) donnée »

Cette définition renferme les aspects les plus importants du texte injonctif/prescriptif et renvoie au schéma cognitif présenté auparavant. Elle fait surgir les actants, l'acte de langage dominant (que nous allons analyser plus loin) et, le plus important, la forme d'algorithme, de programme du texte.

Une autre caractéristique spécifique à ce type de texte est donnée par la macrostructure particulière, une structure sous-jacente qui se manifeste à la surface et organise tous les composants de l'architecture textuelle. Nous allons analyser ce lien spécifique entre la structure sous-jacente et la structure de surface dans le sous-chapitre suivant.

\section{Macrostructure textuelle sous-jacente et structure modulaire de surface}

Dans l'application du concept de macrostructure nous sommes parti des ouvrages de Kintsch et Van Dijk (apud Coirier \& alii 1996) en prenant en compte deux concepts : celui de macrostructure/microstructure et celui de superstructure. Ils définissent la macrostructure comme le sens global du texte (côté sémantique) et la superstructure comme la forme et l'organisation textuelle. Nous avons englobé dans notre concept de macrostructure ces deux aspects. La macrostructure est, donc, un schéma préexistant, présent dans la structure sous-jacente du texte et dans l'esprit des locuteurs et, en même temps, le plan d'organisation textuelle de surface.

Dans le texte prescriptif cette macrostructure a la forme suivante : objectif proposé - stade initial - mode d'action - objectif réalisé

Ou, plus proche du contenu sémantique de ce type de texte : objectif - matériel - suite d'actions à accomplir - résultat 
Ce schéma fonctionne comme organisateur de la surface textuelle est détermine les éléments sémantiques, leur ordre et leurs relations, aussi que l'enchaînement, dans un ordre logique (et chronologique) des opérations.

On peut déjà observer ici une structuration modulaire du texte, chaque partie de cet enchainement constituant un module avec des traits lexico-grammaticaux et modaux spécifiques, reflétés à tous les niveaux de l'organisation textuelle, que nous allons essayer de mettre en évidence dans l'analyse qui suit. À la macrostructure sous-jacente correspondent ainsi des modules d'organisation textuelle de surface. Cette structuration connaît des particularisations spécifiques en fonction du genre textuel de la manière suivante ${ }^{1}$ :

- La recette de cuisine :

obtention d'un plat - ingrédients - opérations culinaires - plat obtenu

- Le mode d'emploi/notice de montage

mise en fonction/montage de l'appareil - parties composantes/ pièces - opérations techniques - mise en fonction/montage

- Le guide touristique

la destination proposée - point de départ - trajet suivi - point d'arrivée

- La règle de jeu

gagner le jeu-le matériau-suite de règles-gagner le jeu

La structure modulaire reflétée au niveau de la surface textuelle est évidente, audelà du spécifique de chaque genre. Elle est organisatrice de la structure compositionnelle du texte et de l'information.

Le premier module représente, d'une manière assez brève, le « sujet » du texte et en même temps place automatiquement le texte dans un genre précis. Il est représenté par le titre, assez souvent accompagné d'une image, surtout dans les recettes de cuisine. Le dernier module, l'objectif atteint, est représenté le plus souvent d'une manière non linguistique, par l'image de l'objet/appareil/produit obtenu ou par une phrase finale du texte qui a le rôle de conclure ce qui était développé dans le texte. L'objectif final est identique avec l'objectif proposé, représentés tous les deux parfois par la même image ce qui crée une circularité et une symétrie parfaite du texte. Assez souvent la réalisation de l'objectif est impliquée dans l'ordre des opérations et s'accomplit par l'achèvement de la dernière opération.

Le corps proprement du texte et formé par les modules qui représentent le matériel (les ingrédients ou les pièces/ les parties d'un appareil) et, surtout, les opérations.

Nous pouvons observer déjà un regroupement sémantique dans ces modules, marqué assez souvent par des titres, tels que : ingrédients, parties composantes, description pour le module des ingrédients et préparation, mise en fonction, utilisation pour celui des opérations. Ce type de présentation favorise une densité informationnelle qui caractérise ce type de texte.

1 Les genres textuels du texte injonctif sont considérés en général : la recette de cuisine, les modes d'emploi, les guides touristiques et les règles de jeu. 
Les modules sont parfois organisés en sous-modules en fonction des dimensions du document qui va d'un schéma d'une page avec quelques explications jusqu'aux dimensions d'une brochure, surtout pour certains modes d'emploi. Dans ce cas, il y a des sous-chapitres qui peuvent reprendre le schéma d'organisation présenté plus haut ou qui peuvent développer seulement un de ces modules. Cet enchaînement modulaire représente aussi l'ordre des actions, ce qui se reflète surtout dans le module des opérations. Ici l'ordre logique et chronologique est très strict. Il y a deux types de progressions possibles : a) d'une opération à l'autre, dans un enchaînement linéaire (surtout dans les modes d'emploi ou les guides touristiques), ou b) par l'obtention des produits intermédiaires, selon les schémas suivants:

a) Ingrédients ou parties composantes - opération ${ }_{1}^{-}$opérations $_{2}$ - opération ${ }_{n}-$ produit final (pour les recettes de cuisine et les modes d'emploi)

objectiffinal à atteindre - objectif $f_{1}$ - chemin (trajet) à parcourir ${ }_{1}$ - objectif - $_{2}$ trajet

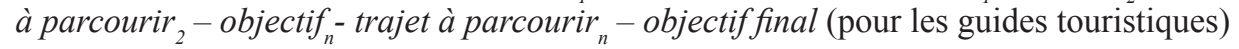

ou bien:

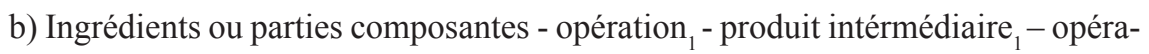
tions $_{2}$ - produit intermédiaire $_{2}-$ opérations $_{n}$ - produit intérmédiaire ${ }_{n}-$ produit final

C'est justement cette organisation qui justifie la dénomination de texte procédu$\mathrm{ral}^{2}$, care il a l'aspect d'un algorithme avec des procédures à suivre pour la réalisation d'un objectif.

Cette présentation textuelle organisée en modules, qui met en évidence, au niveau de surface, la structure sous-jacente du texte, est nouvelle si l'on pense surtout à la recette de cuisine et son développement diachronique sur lequel nous n'allons pas insister maintenant. C'est le résultat d'une expérience de rédaction de la part de l'auteur, mais aussi de la familiarisation du lecteur avec un type de texte, par le contact prolongé avec celui-ci, ce qui favorise la disparition de certains connecteurs textuels et, en général, la disparition de toute information superflue. C'est plutôt sous l'influence des modes d'emploi que la recette prend une forme très organisée avec la mise en évidence des modules qui la composent.

L'organisation en modules a le rôle de mettre en évidence la structure du texte, son organisation en paquets d'informations. De cette façon l'utilisateur se débrouille mieux dans la lecture et le décodage de l'information et dans la mise en pratique des instructions.

La différence entre un texte compact et un texte organisé a été analysée surtout par la linguistique cognitive ${ }^{3}$. Dans ce sens on remarque le fait que la structure modulaire permet, du point de vue cognitif, la réalisation des actions suivantes :

- La mise en relation des informations ;

- Le retour rapide vers les informations antérieures ;

- L'accès rapide à des paquets d'informations.

L'aspect visuel facilite le repérage physique de la structure modulaire du texte. Il y a plusieurs types de marqueurs graphiques qui aident à la mise en relief de la structure modulaire du texte. Ils peuvent être regroupés en deux grandes catégories (Pitar 2015):

2 Pour la différence entre texte injonctif, prescriptif, procédural, voir Pitar 2006, 2014.

3 À voir dans ce sens les articles de Heurley, 2001, Ganier \&alii, 2001, Garcia-Debanc, 2001. 
- Marqueurs qui montrent l'organisation de l'information et la distribution entre les modules ou la séparation de l'information obligatoire de l'information facultative (des espaces qui séparent les modules; le regroupement du texte en colonnes ; l'encadrement du texte (en tableau) ; les titres accompagnés par des images ; la mise en évidence par des couleurs ou des caractères différents);

- Marqueurs qui organisent l'information à l'intérieur d'un module, surtout dans le module le plus important du point de vue de la quantité d'informations, celui des opérations (mise en évidence de chaque étape par les tirets, les points, la numérotation des opérations, des schémas, des dessins, des symboles).

Nous pouvons ainsi observer mieux la coïncidence entre la structure sous-jacente et la structure de surface à tous les niveaux, en tant que structure et en tant que contenu.

\section{Structure modulaire et modalité}

Dans l'analyse antérieure on a pu voir que les modules ont un certain contenu et une certaine fonction et regroupent les informations textuelles en les séparant du point de vue graphique. Nous allons voir maintenant comment ils organisent et distribuent les valeurs modales de ce type de texte.

On ne peut pas séparer les valeurs modales de l'acte de langage spécifique à chaque type de texte. L'acte de langage constitue un des critères principaux dans la classification des textes. Pour le texte prescritif, on considère d'habitude qu'il s'organise autour d'un acte de langage injonctif du type:

\section{Dire / demander à quelqu'un comment/de faire quelque chose}

Celui-ci peut être présenté, d'une manière plus formelle et, en même temps, plus détaillée, sous cette forme :

\section{[a] dit à [b] comment faire pour obtenir [x] en partant de [y]}

Cet acte de langage renferme les éléments composants de la structure sous-jacente - qui sont bien visibles - et fait apparaître les actants. Cette phrase se lit de la manière suivante :

[a] et [b] - auteur et destinataire/ utilisateur

$\mathrm{X}$ - objectif final

$\mathrm{Y}$ - les ingrédients

Comment faire pour- les actions, le chemin actionnel à suivre

Il faut remarquer le lien qui existe toujours entre l'acte de langage et la modalité, car tous les deux expriment la place et le rôle des locuteurs dans le texte et les relations qui s'établissent entre eux et avec le texte.

Il y a, dans le cadre du texte prescriptif, deux mots clé qui nuancent les relations entre les deux actants : dire et faire. Ce sont des verbes hyperonymes, du point de vue sémantique, qui se précisent dans le cas de chaque genre de la manière suivante : 


\section{Faire}

La recette de cuisine - préparer, cuisiner

Le mode d'emploi - employer, assembler

Les guides touristiques - suivre un chemin

La règle de jeu - déplacer les pièces

\section{Dire}

Dans le cadre des actes de paroles directifs, ce verbe connaît les nuances suivantes : ordonner, recommander, expliquer, informer, conseiller qui apparaissent dans tous les genres.

Pourtant, ce qui prédomine dans tous les textes de ce type, c'est l'acte de dire comment faire qui fait surgir une modalité spécifique à ce type de texte : la modalité prescriptive. Elle forme le canevas sur lequel se moulent toutes les autres modalités. Bien que très proche de la valeur modale 0 , elle contient pourtant l'expression d'une position d'autorité ou, au moins, de spécialiste, de la part de l'auteur qui, à partir de cette position, prescrit les actions et leur enchaînement correct pour le succès de leur réalisation. Ce n'est qu'en admettant cette modalité qu'on peut expliquer les mêmes valeurs des modes verbaux différents tels que l'impératif, l'infinitif, l'indicatif présent ou futur qui prédomine dans tous les genres de ce type de texte.

En voici trois séries d'exemples qui prouvent la manifestation de la même valeur prescriptive de ces modes verbaux dans des genres différents du texte procédural: la recette de cuisine, le mode d'emploi et la règle de jeu:

\section{Recette de cuisine}

Rincer les champignons sous le robinet. (infinitif)

Prenez du veau et de la graisse de boeuf et hachez finement tout ensemble. (impératif) Quand la viande est bien cuite on ajoute du vin. (indicatif présent)

Quand la partie que vous voudrez mettre en pâte sera bien lavée vous la larderez de chair d'anguille... vous la piquerez de girofle, vous l'étendrez sur une pâte bien épaisse...(indicatif futur)

\section{Mode d'emploi}

Faire glisser le curseur du volet protège-objectif jusqu'à la position complètement ouverte pour débloquer l'obturateur. (infinitif)

Introduisez l'entraîneur dans le trou du disque et tournez jusqu'en position de verouillage. (impératif)

\section{Règle de jeu}

Posez la carte de départ au milieu de la table, côté recto visible. Ensuite mélangez les cartes de paysage, formez plusieurs piles et posez-les sur la table, face cachée, de manière à ce que chaque joueur puisse piocher facilement des cartes. (impératif)

Mettre les 36 jetons à l'envers et les mélanger. (infinitif)

C'est cette modalité dominante qui justifie la dénomination de texte prescriptif que nous avons choisi dans ce cas et qui fonctionne en parallèle avec les autres dénomina- 
tions - texte injonctif ou texte procédural. Dans le texte prescriptif la structure modulaire spécifique constitue une grille de décodage des différentes nuances modales des verbes et aussi des forces illocutoires.

Chacun des modules présentés contient ses propres modalités, toujours les mêmes. Ainsi le module matériel, que ce soit la liste des ingrédients ou la description/présentation d'un appareil, a une modalité 0, neutre. Le module opérations à suivre renferme, grosso modo, la modalité prescriptive, car l'auteur indique comment faire, quelles sont les opérations et leur ordre. Si l'auteur veut modulariser, nuancer le « comment » faire, cela fait surgir dans le texte des modalisateurs qui sont séparés du texte prescriptif proprement-dit dans un nouveau module dont le rôle et celui d'exprimer les différentes injonctions, un module qui s'ajoute aux modules déjà présentés.

De cette façon nous séparons radicalement cette nuance de l'injonctif de la modalité injonctive proprement-dite. Selon Carmen Stoean (1999), la prescription relève d'un conflit entre le devoir faire du locuteur et le vouloir ne pas faire de l'interlocuteur. Nous considérons que dans ce type de texte ce conflit est annulé par une concordance entre le vouloir des deux actants. Le trait d'autorité du locuteur n'est plus équivalent à une manipulation. Le locuteur est un spécialiste, et son autorité provient des connaissances supplémentaires par rapport à l'interlocuteur/utilisateur qui applique volontiers les indications, les prescriptions du spécialiste. Il s'agit donc d'une collaboration et non pas d'une autorité coercitive.

Nous devons faire encore quelques précisions sur la distinction entre injonctif et prescriptif. Le prescriptif apparaît dans la plupart des ouvrages comme une nuance ou un sous-type des actes de langage directifs et comme une dénomination du type de texte injonctif pour certains auteurs (Adam 2001). Si une phrase déclarative ou assertive est supposée neutre du point de vu pragmatique, de la force illocutoire, la phrase prescriptive se trouve entre une modalité 0 et une injonction très faible (Pitar 2010, 2014). Dans ce texte, écrit par excellence, le prescriptif est le résultat d'une interaction sociale in absentia. L'action prescrite est obligatoire, mais la relation entre les deux locuteurs est plus spéciale. L'auteur ne s'implique pas du point de vue émotionnel, il ne recommande pas, n'ordonne pas et ne demande pas, il prescrit, ce qui instaure une relation entre un spécialiste qui sait et un public qui veut apprendre, tous les deux ayant un même but commun : la réalisation correcte de l'action.

Les rubriques injonctives sont marquées, elles aussi, par des graphismes qui les séparent du reste du texte et qui marquent l'intensité de l'injonction. Parmi ces marqueurs, le plus important est le titre qui nous permet ainsi d'établir l'importance accordée à ces mises en garde, leurs fonctions et leur intensité. Voilà les titres les plus fréquents rangés selon le degré d'intensité de l'injonction :

Conseils

Recommandations

Précautions/ attention

Consignes de sécurité

Avertissements

Interdictions/ danger

Le titre transfère sa valeur injonctive à tout le texte du module qui, de cette façon, n'a pas besoin toujours d'un marquage supplémentaire au niveau des modes verbaux. Pourtant, l'injonction est renforcée par d'autres marqueurs telles que : 
- Le sémantisme des mots : Conseiller, recommander, interdire, faire attention, perdre, éviter, devoir, falloir etc. ;

- La forme négative des verbes;

- Le temps et le mode verbaux : infinitif, impératif (à valeurs injonctives);

- Des symboles !, X 猫

Les mêmes modes et temps verbaux, tels que l'infinitif, l'impératif, l'indicatif présent ou futur, acquièrent, dans le cadre de ce module, une force illocutoire plus forte.

On peut affirmer ainsi que le type de module et l'organisation modulaire confèrent une grille de décodage non seulement sémantique, mais aussi modale.

Voici quelques exemples de tels modules :

Avertissement!

L'étape suivante supprime toutes les données enregistrées dans la mémoire du CSF. Soyez absolument sûr de bien vouloir supprimer toutes les données avant de continuer.

Avertissement de sécurité

N'utilisez l'appareil que pour préparer des repas, jamais en tant que chauffage. Maintenez toujours les enfants éloignés.

Attention!

Toutes les données enregistrées sont perdues si vous enlevez les piles principales et la pile de secours en même temps.

Précautions générales

Assurez-vous que la face positive $(+)$ de chaque pile est dirigée dans le bon sens. Evitez les chocs et les changements subits de température

\section{Recommandations :}

Nous recommandons de ranger les aliments de la manière suivante: Dans le compartiment congelateur: produits surgélés, glaçons, glaces alimentaires. Dans le bac à légumes : légumes, salades, fruits.

Le texte peut contenir un seul module injonctif qui se rapporte au texte en général, ou plusieurs qui peuvent accompagner chaque opération ou peuvent avoir des objectifs différents. Le nombre et le contenu des rubriques injonctives sont différents en fonction du genre. Dans la recette de cuisine ce sont plutôt les conseils qui font l'objet d'un module séparé. En revanche, les modes d'emploi et les notices de montage sont les plus riches en avertissements et mises en garde de toutes sortes, car les opérations à accomplir supposent des précautions supplémentaires. Si la préparation d'un plat est une activité quotidienne à la portée de tout le monde, la manipulation d'un appareil, parfois inconnu, suppose une attention particulière de la part de l'utilisateur et des précautions à prendre quant au contenu du texte de la part de l'auteur/ producteur.

Pour résumer, on peut dire que dans un texte prescriptif organisé il y a trois grandes parties avec des modalités différentes :

- énumération des produits ou des pièces composantes (description de l'appareil) - modalité 0 ; 
- indication des opérations -modalité prescriptive;

- des précautions et des avertissements - modalités injonctives de degrés de forces et valeurs différents.

Malgré l'importance de l'information qui vise la mise en garde du lecteur, le texte disparait parfois en faveur de l'image ou des symboles. On peut considérer que certains symboles sont plus aptes à exprimer le danger possible. C'est pourquoi ils accompagnent le texte ou même le remplace.

\section{Conclusion}

Comme on l'a pu observer, les genres du texte prescriptif sont des genres très organisés du point de vue de l'architecture textuelle. Cette structuration est due à un schéma sous-jacent organisateur de la surface textuelle qui soutient - d'une manière plus ou moins consciente de la part des locuteurs - tous les types de texte. La structure de surface, très organisée et très marquée dans le cas du texte prescriptif, est due en premier lieu au caractère pratique du texte, mais aussi à son prolifération et multiplication. Comme intermédiaire entre deux actions, le texte a comme but une présentation très claire des actions à accomplir et des précautions à prendre. À cette fin, tous les moyens sont employés : texte, images, dessins, schéma, place dans la page etc. Il y a, d'une part, l'effort de l'auteur de faire comprendre le message du texte, car aucune interprétation subjective n'est pas admissible, d'où un sens univoque, l'élimination de la polysémie etc. D'autre part, le destinataire/utilisateur doit savoir se débrouiller dans le texte et décoder d'une manière correcte les informations transmises. Chaque module lui transmet un certain type d'information aussi que la manière de décodage de cette information. Il s'agit, des deux côtés, d'une expérience de rédaction/ lecture du texte. Comme on l'a vu, l'organisation modulaire du texte est l'expression de cette expérience et joue un rôle essentiel dans la compréhension.

En guise de point final, nous allons présenter le rôle du schéma organisateur du texte selon les chercheurs de la linguistique cognitive (Coirier et alii., 1996). Celui-ci :

- assure la cohérence du texte et la réalisation des inférences nécessaires pour le décodage de l'information;

- établit le degré d'importance de l'information;

- permet au lecteur d'anticiper l'organisation ultérieure du texte ;

- offre un repérage rapide des paquets d'information.

Le texte prescriptif continue d'ailleurs de former l'objet d'étude des linguistes, surtout du point de vue cognitif.

\section{Références:}

Adam, J.M. (1992). Les textes: Types et Prototypes. Récit, description, argumentation, explication et dialogue, Paris, Nathan.

Adam, J.M. (2001). Entre conseil et consigne: les genres d'incitation à l'action. In : Pratiques 11/112, 7-38.

Bronckart J.-P. (1997). Activité langagière, textes et discours, Lausanne-Paris, Delachaux et Niestlé. 
Coirier P., Gaonac'h D., Passerault J.-M. (1996). Psycholinguistique textuelle, Approche cognitive de la compréhension et de la production des textes, Armand Colin, Paris.

Ganier, F. et alii. (2001). Discours procédural et activités mentales : de la compréhension d'instructions complexes à la planification de l'action. In : Langages 141, 47-64.

Garcia-Debanc, C. (2001). L'étude des discours procéduraux aujourd'hui : travaux linguistiques et psycholinguistiques. In : Langages 141, 3-18.

Heurley, L. (2001). Du langage à l'action : le fonctionnement des textes procéduraux. In : Langages 141, 64-79.

Pitar, M. (2006). Text şi textualitate: două concepte definitorii ale gramaticii textuale. In: Comunicare profesională şi traductologie, Lucrările conferinței internaționale, 25-26 Septembrie 2003, Timişoara, România, Ed. Orizonturi Universitare, Timişoara, 2004, 141-147.

Pitar, M. (2010). Type de texte et modalité. Le cas du texte injonctif. In : Actes $d u X X V e$ Congrès International de Linguistique et de Philologie Romanes (Innsbruck 3-8 septembrie 2007), tome V, Ed. De Gruyter, Berlin, 503-513.

Pitar, M. (2014), Textul injonctiv. Repere teoretice, Ediţia a II-a revăzută şi adăugită, Timişoara, Excelsior Art.

Pitar, M. (2015). Marqueurs de décodage dans la compréhension des genres textuels. Le cas des modes d'emploi. In : Discours en présence. Hommage à Liana Pop, Presa universitară clujeană, Cluj, 2015, 109-123. de mâine».

Stoean, C.S. (1999), Temps, modes et actes de paroles, Bucureşti, Ed Fundaţiei « România

\section{Мариана Питар}

\section{МОДУЛАРНА СТРУКТУРА ПРЕСКРИПТИВНОГ ТЕКСТА - УЛОГА И МОДАЛИТЕТИ}

Сажетак: Прескриптиван текст, назван и текст-налог или процедурални, у зависности од прихваћене перспективе, садржи неколико познатих жанрова, укључујући и кухињске рецепте и начин употребе.

Имајући веома значајну историјску подлогу, кухињски рецепт прелази из компактног текста, помешан са наративним елементима, у боље структуриран текст, са посебним деловима. Новија употреба дошла је веома брзо до чисте структуре, организоване у модулима, тако да се информација преноси путем два семиотичка кода: текста и слике. Циљ овог рада јесте да представи овај тип организације модуларног текста и његовог дејства на структуру текста и укључених модула. Користећи пример две жанровске методе, анализираћемо композициону структуру текста, као структуре заједничке свим жанровима прескриптивног текста:

Предложени циљ - почетно стање - процес - крај - достигнућа.

Ова текстуална макроструктура организује текст у модулима, сваки са специфичном улогом у семантици, синтаксици и композицији, изражавајући јасно садржину текста.

Занимљива је чињеница да ова структура одређује избор одређених начина, различитих за сваки модул, који је изражен кроз специфичне језичке нивое (глаголски начин, семантичко значење речи итд.) на екстрајезички (слике, симболи, позиција текста на страни итд.) Ми ћемо проучавати ове знаке у вези са модуларном структуром текста. 